\title{
Application of Graph Entropy in CRISPR and Repeats Detection in DNA Sequences
}

\author{
Dipendra C. Sengupta, Jharna D. Sengupta \\ Department of Mathematics \& Computer Science, Elizabeth City State University, Elizabeth City, NC, USA \\ Email: dcsengupta@ecsu.edu, jdsengupta@ecsu.edu
}

How to cite this paper: Sengupta, D.C. and Sengupta, J.D. (2016) Application of Graph Entropy in CRISPR and Repeats Detection in DNA Sequences. Computational Molecular Bioscience, 6, 41-51.

http://dx.doi.org/10.4236/cmb.2016.63004

Received: January 24, 2016

Accepted: October 23, 2016

Published: October 26, 2016

Copyright $\odot 2016$ by authors and Scientific Research Publishing Inc. This work is licensed under the Creative Commons Attribution International License (CC BY 4.0).

http://creativecommons.org/licenses/by/4.0/ (c) (i) Open Access

\begin{abstract}
We analyzed DNA sequences using a new measure of entropy. The general aim was to analyze DNA sequences and find interesting sections of a genome using a new formulation of Shannon like entropy. We developed this new measure of entropy for any non-trivial graph or, more broadly, for any square matrix whose non-zero elements represent probabilistic weights assigned to connections or transitions between pairs of vertices. The new measure is called the graph entropy and it quantifies the aggregate indeterminacy effected by the variety of unique walks that exist between each pair of vertices. The new tool is shown to be uniquely capable of revealing CRISPR regions in bacterial genomes and to identify Tandem repeats and Direct repeats of genome. We have done experiment on 26 species and found many tandem repeats and direct repeats (CRISPR for bacteria or archaea). There are several existing separate CRISPR or Tandem finder tools but our entropy can find both of these features if present in genome.
\end{abstract}

\section{Keywords}

CRISPR, Graph Entropy, Tandem Repeats, DNA Sequences

\section{Introduction}

Deciphering the enormously long nucleotide sequences that are being uncovered in the human genome is one of the major challenges in our days. Along with serious ethical issues, we encounter a series of tremendously hard scientific problems. These problems mainly arise from the fact that although sequencing techniques are almost completely automatic controlled the analysis of the sequenced data is not. Hence, the major goal of the Human Genome Project is the extraction of biologically and medically relevant information from almost automatically sequenced DNA and RNA molecules. In prin- 
ciple, biochemical methods are able to do this job, but since they are extremely expensive and time consuming, there is a high demand for alternative approaches to extract the information hidden in genome [1]. In this situation, concepts and techniques from information theory turned out to be welcoming tools to handle the problem of extracting valuable information from biosequences such as DNA, RNA, or amino acid chains. The main goal of our work is the presentation of a concept and method derived from information theory that will apply to problems of analysis of DNA.

The motivation for this study is to analyze DNA sequences to determine interesting sections of genome that has repeating features using information theory tool.

In many organisms, the genomic DNA is highly repetitive accounting for close to $5 \%$ of the genome size [2] [3]. Repetitive DNA sequences are a major component of eukaryotic genomes and may account for up to $90 \%$ of the genome size [4]. The human genome itself has over two-thirds of the sequence consisting of repetitive elements [5]. The identification of repeats has proven to be of significance, as they provide insight into the functional and evolutionary roles of various organisms [6] [7] [8] [9] [10].

In our study we also focus on a family of repeats known as Clustered Regularly Inter Spaced Palindromic Repeats (CRISPRs) [11]. CRISPRs have attracted a great deal of interest recently in genome editing [12]. CRISPRs have been found only in the genomes of prokaryotes and are composed of short direct repeats currently known to range in sizes from $21-47$ base pairs. This family of repeats is unique in that they are interspaced by non-repeating sequences of similar size, called spacers. CRISPRs were found in approximately $40 \%$ of bacterial genome investigated [13].

Several software applications are available for identifying various form of repeats in [14] [15] [16].

\section{Graph Entropy Algorithm}

A graph is an object that consists of a non-empty set of vertices and another set of edges. Vertices are often called nodes, and edges are referred as connections. The set of edges may be empty, in which case the graph is just a collection of points.

We say that two vertices $i$ and $j$ of a directed graph are connected if there is an edge from $i$ to $j$ or from $j$ and $i$. Suppose we are given a directed graph with $\mathrm{n}$ vertices. We construct an $n \times n$ adjacency matrix A associated to it as follows: if there is an edge from vertex $i$ to vertex $j$, we put 1 as the entry on row $\mathrm{i}$, column $\mathrm{j}$ of the matrix $A$; if there is no edge, we put 0 .

If one can walk from vertex $i$ to vertex $j$ along the edges of the graph then we say that there is a path from $i$ to $j$. If we walked on k edges, then the path has length $k$. For matrices, we denote by $A^{k}$ the matrix obtained by multiplying $A$ with itself $k$ times. The entry on row $i$, column $j$ of $A^{2}$ corresponds to the number of paths of length 2 from vertex $i$ to vertex $j$ in the graph.

Let us consider a directed graph and a positive integer $k$. Then the number of directed walks from vertex $i$ to vertex $j$ of length $k$ is the entry on row $i$ and column $j$ of the matrix $A^{k}$, where $A$ is the adjacency matrix. 
In this section, we will discuss entropy of such adjacency matrix $A$. Let $\left\{v_{1}, v_{2}, \ldots, v_{n}\right\}$ be a set of vertices of a direct graph. Let $A=\left(a_{i j}\right)$ be the $n \times n$ adjacency matrix with at least one positive element.

Let $\sum_{i, j}^{n} a_{i j}=N$. Let $P=\left(P_{i j}\right)$ be the matrix such that $P=\frac{A}{N}$. Hence $\sum_{i, j}^{n} p_{i j}=1$. $p_{i j}$ is the probability of having a path from vertex $v_{i}$ to vertex $v_{j}$. Adding all elements of each row of $\mathrm{P}$ and placing them on the diagonal, we form a diagonal matrix

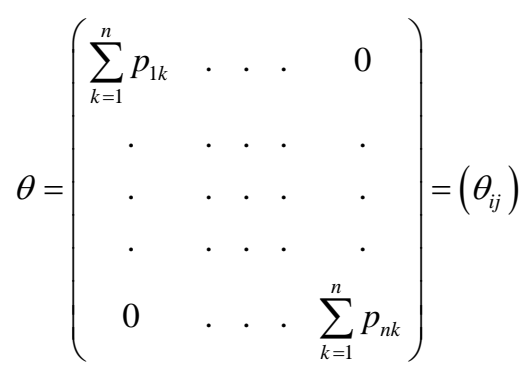

$1-\theta_{j j}=\left(1-\sum_{k=1}^{n} p_{j k}\right)$ is the probability for a randomly generated path to end at the vertex $v_{j}$. Let $Q_{i j}(l)$ be the probability for generating a path of length $l$ that begins at $v_{i}$ and ends at $v_{j}$ for any integer 1 . For example, we have $Q_{i j}(1)=p_{i j}\left(1-\sum_{k=1}^{n} p_{j k}\right)$ and $Q_{i j}(2)=\left(p_{i 1}, p_{i 2}, \ldots, p_{i n}\right)\left(\begin{array}{c}p_{1 j} \\ \cdot \\ \cdot \\ \cdot \\ p_{n j}\end{array}\right)\left(1-\sum_{k=1}^{n} p_{j k}\right)$. Let $Q_{l}$ be the matrix whose $i j$ element is $Q_{i j}(l)$. Then we have $Q_{l}=P^{I}(I-\theta)$. Finally, we define the asymptotic walk matrix $\Omega=\left(\Omega_{i j}\right)$ as $\Omega \equiv \sum_{l=1}^{\infty} Q_{l}$, Where $\Omega_{i j}$ is the probability for generating a walk of any length from $v_{i}$ to $v_{j}$.

Note that $\sum_{i, j} \Omega_{i j}=1 . \quad \Omega \equiv \sum_{l=1}^{\infty} Q_{l}=P(I-\theta)+P^{2}(I-\theta)+\cdots$

We noticed that the sum of all entrees of the matrix $P^{\lambda}(Q-P)$, for any integer $\lambda$, is 0 . Since sum of all entrees of $\mathrm{P}$ is 1 , sum of all entrees of $\Omega$ is also1. We therefore define the asymptotic entropy

$$
\mathrm{H}(P)=-\sum_{i, j} \Omega_{i j} \log \left(\Omega_{i j}\right)
$$

where $\Omega_{i j} \log \left(\Omega_{i j}\right)$ is defined to be 0 for $\Omega_{i j}=0$. This can also be called the graph entropy of the graph or entropy of the adjacency matrix $A$. For illustration, Let us consider a short sequence:

\section{ATGCCTGATGCGACGC}

Taking 2-letter nodes with one overlap, we can create a graph as following: 


$$
\begin{aligned}
& A T \rightarrow T G \rightarrow G C \rightarrow C C \rightarrow C T \rightarrow T G \rightarrow G A \rightarrow \\
& A T \rightarrow T G \rightarrow G C \rightarrow C G \rightarrow G A \rightarrow A C \rightarrow C G \rightarrow G C
\end{aligned}
$$

We draw a graph as in the Figure 1.

For our sequence, graph entropy

$$
\mathrm{H}(P)=-\sum_{i, j} \Omega_{i j} \log \left(\Omega_{i j}\right) \approx 2.9614
$$

\section{Results}

We have downloaded wide range of genome data, eukaryotes (animals, plants, insects, fungus) and prokaryotes (bacteria, archaea) from Gen Bank:

ftp://ftp.ncbi.nlm.nih.gov/genomes/.

We have implemented the Graph Entropy Algorithm in MATLAB platform and converted data to MATLAB format. Then we have computed graph entropy using our Graph Entropy Algorithm by scanning the data with a typical sample size of 512 base pairs (bp) and step size of 10 bp taking 3 nodes with 1 overlap. We have drawn graphs of entropy versus genome length of Acidovorax bacteria in Figure 2, Salmonella-Typhi CT18 bacteria in Figure 3, Caldicellulosiruptor Kristianssonii bacteria in Figure 4 and Human Chromosome-21 in Figure 5. We have studied the intervals visually where entropy was low and found some repetitive pattern in the sequence. Once we have a string of repetitive pattern we used MATLAB "strfind" command to find out exact positions of the repetitive patterns. We have included few examples in this paper, only the ones we thought important.

In Figure 2 we looked at the lowest drop of entropy which is at: $x$ : genome length = $871,100, y$. entropy $=4.088$. We took an interval $(871,000,871,600)$ around the lowest drop $x=871,100$.

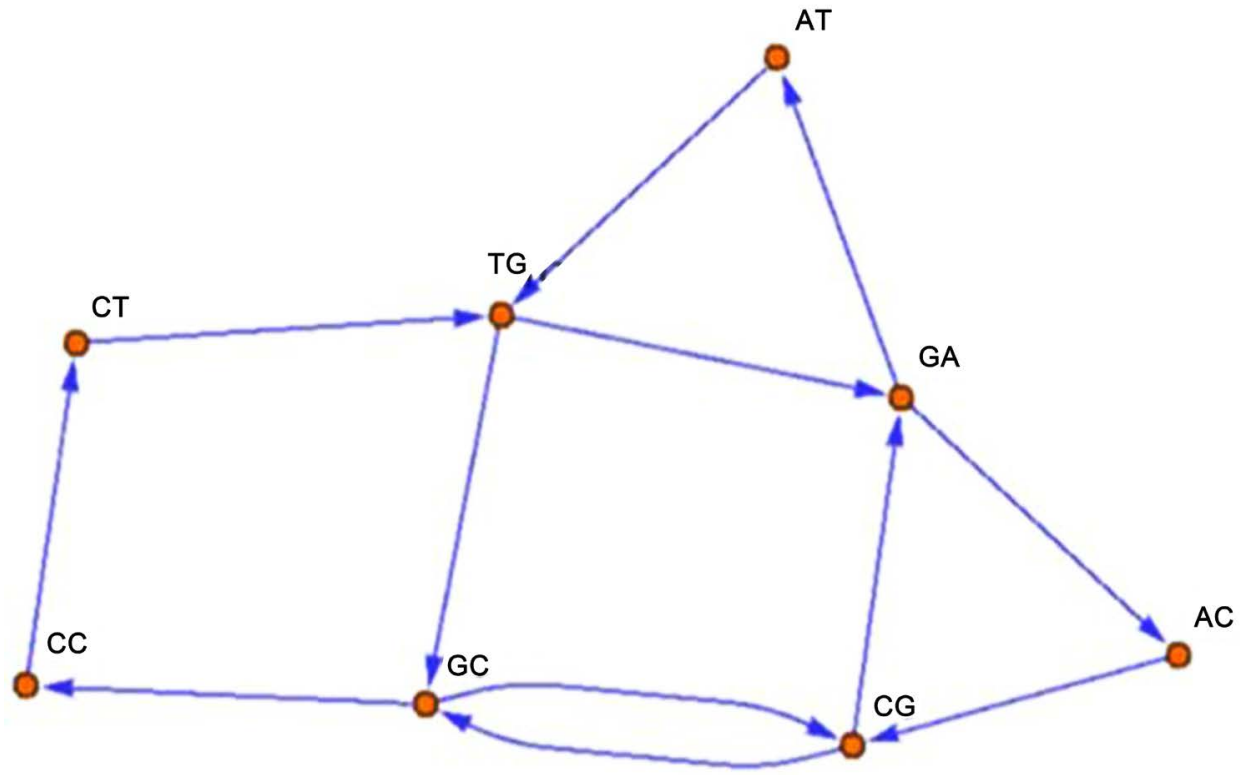

Figure 1. Example of DNA graph. 


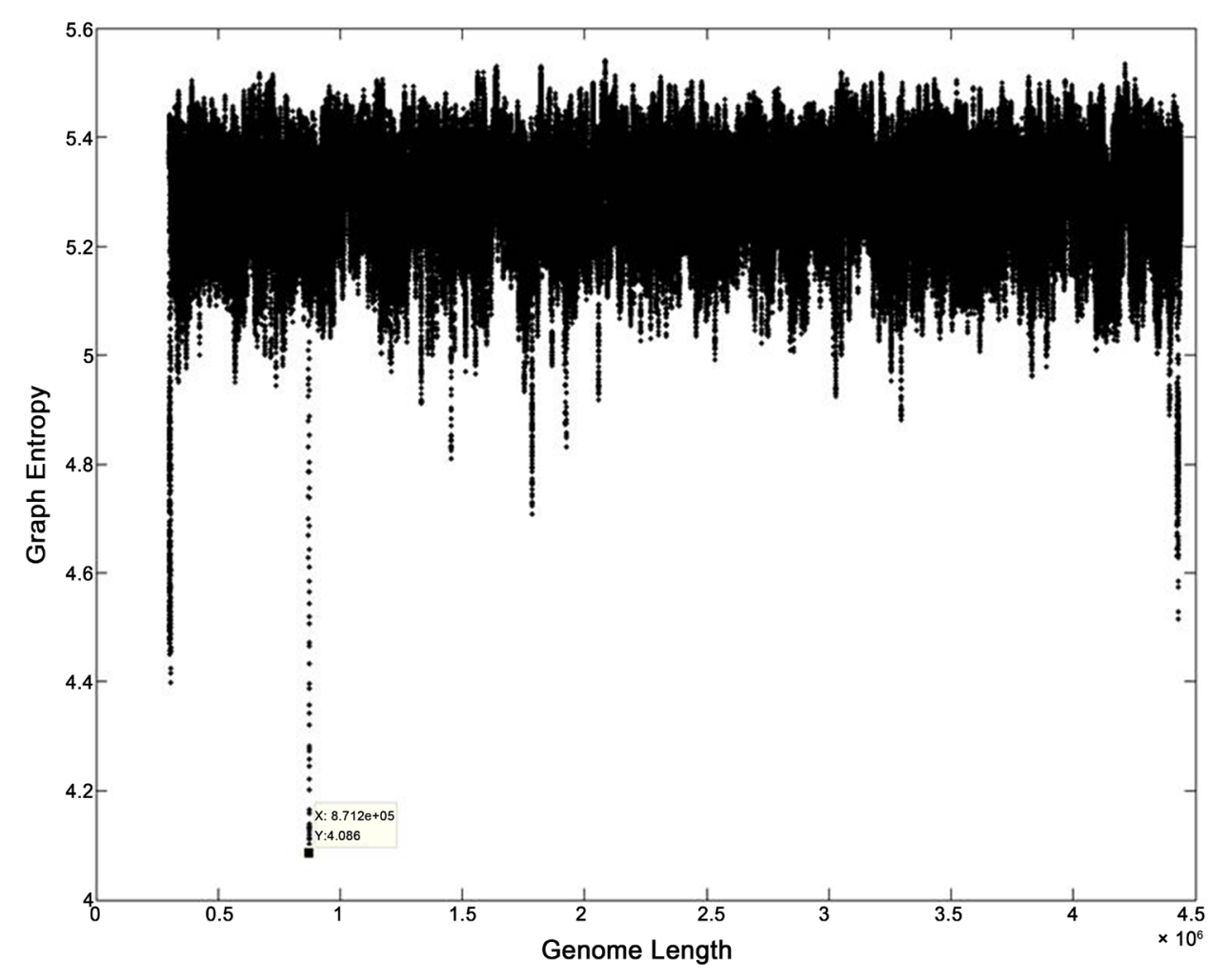

Figure 2. Acidovorax (bacteria) genome length vs entropy.

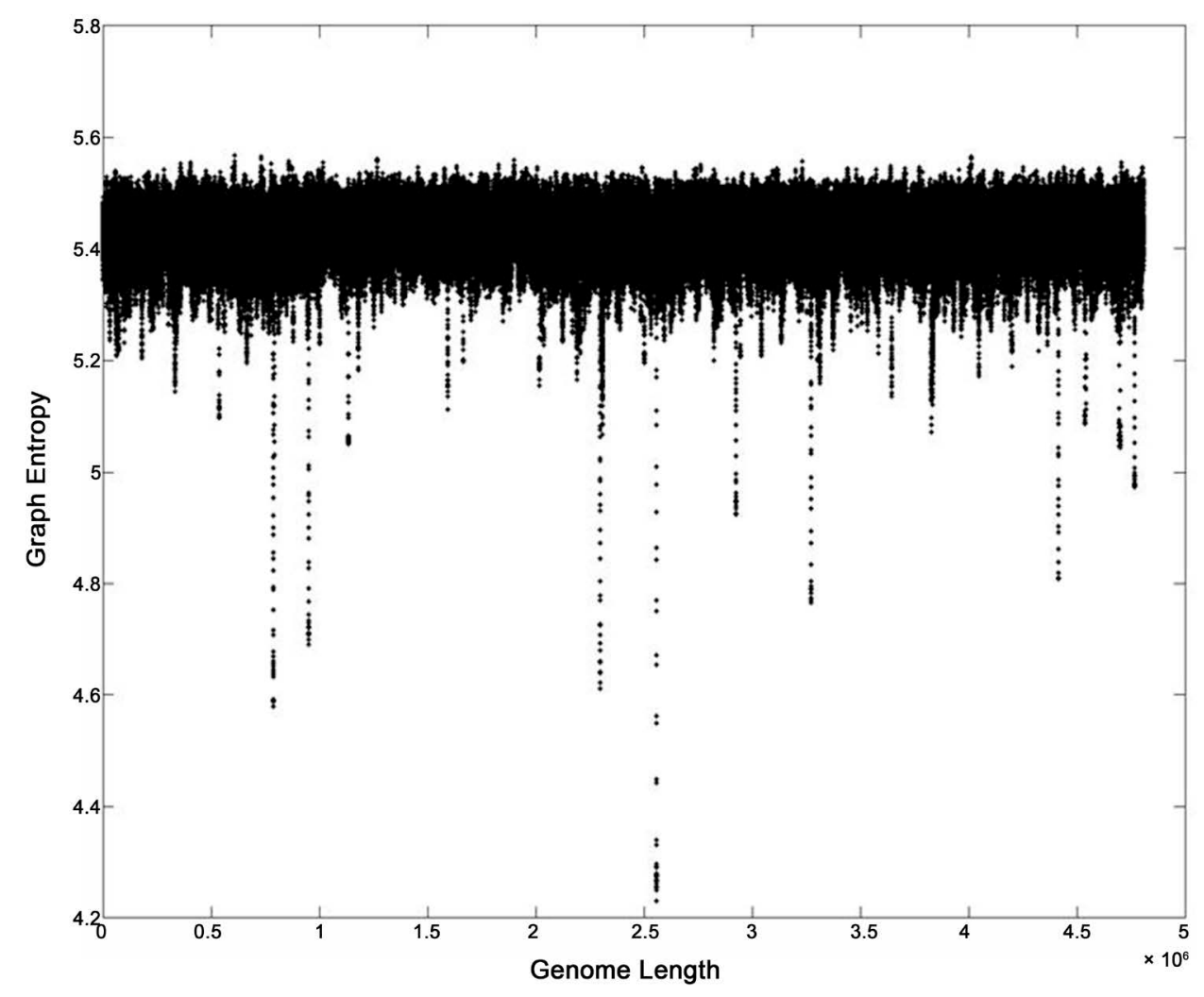

Figure 3. Salmonella-typhi CT18 (bacteria). 


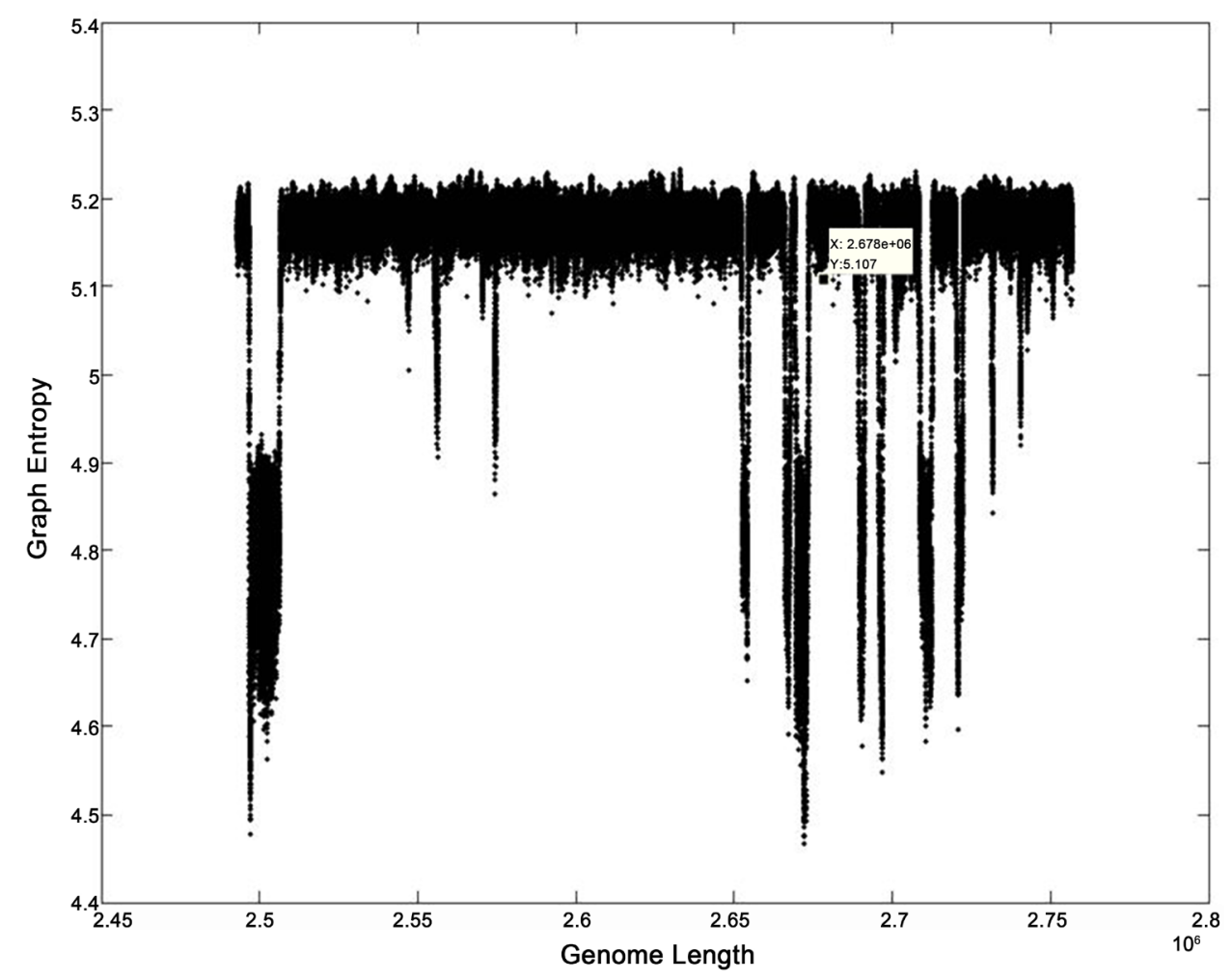

Figure 4. Caldicellulosiruptor kristianssonii (bacteria).

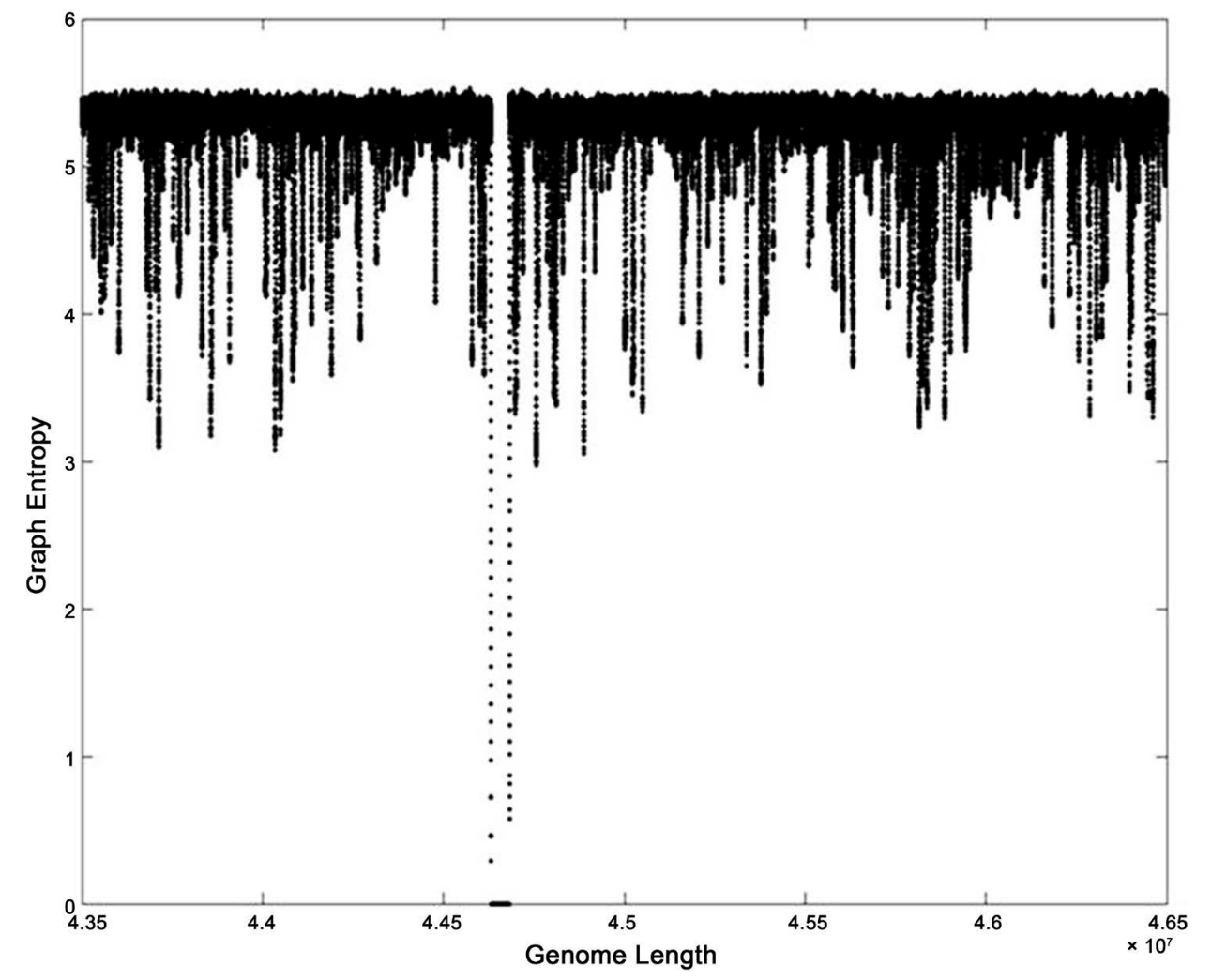

Figure 5. Human chromosome-21. 
The following is the sequence in the interval taken. The colored string is repeating.

ATAAAAAAACCCGGTGCATGCACCGGGTGGGACCAGCCCCGCGGGCGG GGCGGCTGGCTGCTGTCGTCGCTCAGGGCTTGGTGCCCGTCGGGAAGGG CCATGCGGCCTGCGGGTTCAGCGTGGTCTGTGCTGCGGGTGCAGGCGCA GGGGCAGAGGCCTTGGAGGCCGCCTTTTTCGGGGCAGCCTTCTTCGGTG CAGCGGCCTTGGTCGTGCCGGTGGCCTTCTTCGCCGGTGCAGCTGCCTTC TTGGTGGAGGCTGCGGCCTTCTTTGCCGGTGCAGCTGCCTTCTTGGCGG GGGCTGCGGCCTTCTTCGCCGGTGCAGCTGCCTTCTTGGCGGGGGCTGC GGCCTTCTTTGCCGGTGCAGCTGCCTTCTTGGCAGGAGCTGCGGCCTTCT TTGCCGGTGCAGCTGCCTTCTTGGCGGGGGCTGCGGCCTTCTTTGCCGGT GCAGCTGCCTTCTTGGCAGGAGCTGCGGCCTTCTTTGCCGGTGCAGCTG CCTTCTTGGCAGGAGCTGCGGCCTTCTTTGCCGGTGCAGCTGCCTTCTTG GCGGGGGCTGCAGCCTTCTTCGCCGGAGCGGCCTTCTTCGTCGTGGCGG CGGCCTTCTT

Strfind(g,'GCCGGTGCAGCTGCCTTCTTGG') command gave us the following positions of those repeats in the sequence.

$\begin{array}{llllllll}871227 & 871269 & 871311 & 871353 & 871395 & 871437 & 871479 & 871521\end{array}$

The spacers are almost identical. These are tandem repeats.

Similarly in the Figure 3 we looked at the lowest drop of entropy which is at $x=$ $2926000 y=4.923$.

We looked at the DNA sequence in the interval (2926000:2926650) around $x=$ 2926000. The following is the sequence in the interval taken. The colored string is repeating.

AAAAATGCATCCTTCCCGAACGGCAATAGCTGGCACGACGTACGGCTTG ATAATCAACAGCATATAGACAAGGCGCTGCCAGGGCGGATTGAGCGCCG TAGCCGCGATGTAGTGCGGATAATGCTGCCGTTGGTAAAAGAGCTGGCG AAGGCGGAAAAAACGTCCTGATATGCTGGTGAAACGTGTTTATCCCCGC TGGCGCGGGGAACACGGACAGCAACCCGTGTCGGATATCAGACAGATCG GTTTATCCCCGCTGGCGCGGGGAACACACGCGAATCGCCAATCGCCGCC GCGTGAATTGCGGTTTATCCCCGCTGGCGCGGGGAACACCCACGATGTA TGCCGACCGTGATTTTTACCGCCGGTTTATCCCCGCTGGCGCGGGGAAC ACAGATACGCCTTTACGTCGCCCTCTTTGGCGCGCGGTTTATCCCCGCTG GCGCGGGGAACACTAAAACACCGGTTGCGCAACCTCCGCGGGGATCGGT TTATCCCCGCTGGCGCGGGGATCGGTTTATCCCCGCTGGCGCGGGGATC GGTTTATCCCCGCTGGCGCGGGGAACACTCTAAATCTACCCAATTGAATT TAAATACTTTTTTAGCGCACAAAAAACCCACCAACTTTTCCTAATTTTTA AAGATCTCTAA

We used strfind(g,'CGGTTTATCCCCGCTGGCGCGGGGAACAC') in MatLab and found more repeats outside the interval.

$29262432926304 \quad 2926365 \quad 2926426 \quad 29265392943184$ (does not be- 
long to this region).

length('CGGTTTATCCCCGCTGGCGCGGGGAACAC')=29

strfind(g,'GTGTTTATCCCCGCTGGCGCGGGGAACAC'): 2926182

strfind(g,'CGGTTTATCCCCGCTGGCGCGGGGATCGG') 29264872926513.

Starts: 2926182 Ends: 2926567.

In the interval $(2926182,2926513)$ we find three strings differing by 2 to 4 letters.

These repeats are called CRISPR. This is only CRISPR so far known for this strain of the bacteria.

Again, we studied the pattern of the DNA sequence of Caldicellulosiruptor Kristianssonii (Bacteria) in intervals around the points of low entropy and found repetitive patterns. In Figure 4, we considered the drop at $\mathrm{x}=2,672,000, \mathrm{y}=4.46$. Following is the sequence in the interval $(2671900,2672600)$ around this drop. The repeats are shown in red color.

TATTGCAATTATTGTCCTATGCACAGAGTTTGTAGCCTTCCCGTTGGGGA TTGAAACATAGATTTCATTTCGCAGCCAATAGAGCGGTTTATAGTTTGTA GCCTTCCCGTTGGGGATTGAAACCTCAATTTCTGTTTCTCTTTTCTCAATT ATTCTTGAGTTTGTAGCCTTCCCGTTGGGGATTGAAACTATAATAGCCCA TTCATCAAAAACTTTTTCATCGAAGTTTGTAGCCTTCCCGTTGGGGATTG AAACTATAATAGCCCATTCATCAAAAACTTTTTCATCGAAGTTTGTAGCC TTCCCGTTGGGGATTGAAACCACAAAATTATAGTTTGGCGCAATGTAAA CACGAACAGTTTGTAGCCTTCCCGTTGGGGATTGAAACTCTATGTCTTCT TCAAGATACATATCGAGCAGCTTATTGTTTGTAGCCTTCCCGTTGGGGAT TGAAACATACTTTTTTTCTCACGGTCTGTATGGCCTGTTCAGT

We notice repeats and use Matlab to find the exact locations of that string. strfind(g,'GTTTGTAGCCTTCCCGTTGGGGATTGAAAC')

Columns 1 through 61

$\begin{array}{llllllll}2666352 & 2666419 & 2666484 & 2666551 & 2666616 & 2666682 & 2666748 & 2666813 \\ 2666879 & 2666947 & 2667014 & 2667081 & 2667147 & 2667213 & 2667278 & 2667344 \\ 2667410 & 2667476 & 2667544 & 2667611 & 2667676 & 2667741 & 2667805 & 2667872 \\ 2667939 & 2670817 & 2670882 & 2670949 & 2671016 & 2671081 & 2671147 & 2671214 \\ 2671279 & 2671345 & 2671411 & 2671476 & 2671544 & 2671610 & 2671675 & 2671740 \\ 2671805 & 2671871 & 2671936 & 2672001 & 2672070 & 2672135 & 2672201 & 2672267 \\ 2672333 & 2672399 & 2672466 & 2672534 & 2672599 & 2672666 & 2672733 & 2672798 \\ 2672864 & 2672930 & 2672996 & 2673523 & 2673590 & & & \end{array}$

Length ('GTTTGTAGCCTTCCCGTTGGGGATTGAAAC') $=30$

61 repeats of length of 30 , unique spacers

Starts: 2666352 Ends: 2673620 Period: 65/66/67 Total Length $=7268$

These repeats are CRISPR.

In Figure 5, we considered the drop at $\mathrm{x}=44010000, \mathrm{y}=4.13$ and the interval (44009900, 44010500). Following is the sequence of Human Chromosome-21in that interval. We also found repeats. 


\begin{abstract}
CCGTTTATATCCACGCAGGCGTTTCCCCTTACCTGCACCGAGCCTCCATT
CCCGTTTATATCCACGCAGGCGTTTCCCCTTACCTGCACCGAGCCTCCCG

CCCCGTTTACATCCACGCAGGCGTTTCCCCTTACCTGCACCGAGCCTCCA

TTCCCGTTTATATCCACGCAGGCGTTTCCCCTTACCTGCACCGAGCCTCC

CGCCCCGTTTACATCCACGCAGGCGTTTCCCCTTACCTGCACCGAGCCTC

CATTCCCGTTTATATCCACGCAGGCGTTTCCCCTTACCTGCACCGAGCCT

CCCGCCCCGTTTACATCCACGCAGGCGTTTCCCCTTACCTGCACCGAGCC

TCCATTCCCGTTTATATCCACGCAGGCGTTTCCCCTTACCTGCACCGAGC

CTCCATTCCCGTTTATATCCACGCAGGCGTTTCCCCTTACCTGCACCGAG

CCTCCATTCCCGTTTATATCCACGCAGGCGTTTCCCCTTACCTGCACCGA

GCCTCCCGCCCCGTTTATATCCACGCAGGCGTTTCCCCTTACCTGCACCG

GGCCTGCCGCCCCGTTTACATCCACGCATGCGTTTCCCCTTACCTGCACT

G
\end{abstract}
strfind(g,'TTTCCCCTTACCTGCACCGAGCCTCCATTCCCGTTTATATCCACGCA GGCG')
Columns 1 through 18

$\begin{array}{llllllll}44007626 & 44008952 & 44009105 & 44009156 & 44009258 & 44009360 & 44009462\end{array}$
$\begin{array}{llllllll}44009513 & 44009615 & 44009717 & 44009819 & 44009870 & 44009921 & 44010023\end{array}$
$44010125 \quad 44010227 \quad 44010278 \quad 44010329$

The spacers are almost identical with this string except 4 letters (in purple). We also find the spacer string.

Strfind(g,"TTTCCCCTTACCTGCACCGAGCCTCCCGCCCCGTTTACATCCAC GCAGGCG”).

Columns 1 through 23

$\begin{array}{llllllll}44007575 & 44007677 & 44007728 & 44007779 & 44007830 & 44007881 & 44007983 \\ 44008034 & 44008289 & 44008493 & 44008646 & 44008697 & 44008799 & 44008850 \\ 44008901 & 44009207 & 44009309 & 44009564 & 44009666 & 44009768 & 44009972 \\ 44010074 & 44010176 & & & & & \end{array}$

This is a repeat of a string without any gap in the region $(44007575,44010329)$.

\title{
Discussion
}

The importance of identifying repetitive sequences is clear; however, the considerable size of many genomes makes fast and efficient repeat detection very challenging. In this paper, we have presented a new algorithm for finding repeats in DNA sequences. The algorithm is based on our new measure of entropy for any non-trivial graph. In [15], an algorithm were presented for finding tandem repeats in DNA sequences based on the detection of k-tuple matches. It uses a probabilistic model of tandem repeats and a collection of statistical criteria based on that model. Whereas in [14] and [16] a new tool was introduced for the automatic detection of CRISPR elements in genome. The main advantage of our tool is it will detect both tandem repeats and CRISPR or any other repeats. The main disadvantage of our tool is lack of complete automation and hence it is less efficient compared to the other tools. Our detection technique convert sequences to 
an alternative representation (namely, graph as it is given in [17]) in an attempt to make analysis more efficient. Future research plans are to modify the presented algorithm so that it is also able to identify repeats efficiently. Our code will be available to the reader upon request through email to one of the authors.

\section{Conclusions}

We have studied the following species:

Eukaryotes: Homo sapiens chromosome 19 \& 21, Anopheles gambiae, Caenorhabditis elegans, Plasmodium falciparum Saccharomyces cerevisiae.

Prokaryotes: Acidovorax, Ammonifex, Caldicellulosiruptor kristjanssonii, E.Coli, Salmonella Typhi, Listeria Monocyto genes, Bacillus clausii KSM, Chlamydia muridarum Nigg, Cyanobacterium aponinum, Gluconacetobacter diazotrophicus, Haemophilus influenzae R2866, Mycobacterium tuberculosis, Mycoplasma genitalium, Neisseria meningitidis, Streptococcus pneumoniae, Thermosipho africanus, Truepera radiovictrix (Bacteria), A. fulgidus (Archaea).

Viruses: HIV, Hepatitis B. After analyzing the DNA sequence at the points of low entropy for all these species, we conclude that low entropy in a genome graph corresponds to high repeatability in the sequence. These repeats can be classified as CRISPR or Tandem Repeats or something else.

\section{Acknowledgements}

This paper was written while two authors were Summer Faculty Fellow in SPAWARS YSCEN Atlantic, Charleston, SC funded by Office of Naval Research. Authors are thankful to their mentor for his assistance in the work.

\section{References}

[1] Grosse, I. (2000) Applications of Statistical Physics and Information Theory to the Analysis of DNA Sequences. Ph.D. Dissertation, Boston University, MA.

[2] Ussery, D.W., Binnewies, T.T., Gouveia-Oliveira, R., Jarmer, H. and Hallin, P.F. (2004) Genome Update: DNA Repeats in Bacterial Genomes. Microbilogy, 150, 3519-3521. http://dx.doi.org/10.1099/mic.0.27628-0

[3] Hofnung, M. and Shapiro, J. (1999) On Bacterial Repeats. Research in Microbiology (Special November-December Double Issue on Bacterial Repeats), 150, 577-578.

[4] Mehrotra, S. and Goyal, V. (2014) Repetitive Sequences in Plant Nuclear DNA: Types, Distribution, Evolution and Function. Genomics Protemics Bioinformatics, 12, 164-171. http://dx.doi.org/10.1016/j.gpb.2014.07.003

[5] de Koning, A.P.J., Gu, W., Castoe, T.A., Batzer, M.A. and Pollock, D.D. (2011) Repetitive Elements May Comprise Over Two-Thirds of the Human Genome. PLoS Genet, 7, Article ID: e1002384. http://dx.doi.org/10.1371/journal.pgen.1002384

[6] Achaz, G., Coissac, E., Netter, P. and Rocha, E.P. (2003) Associations between Inverted Repeats and the Structural Evolution of Bacterial Genomes. Genetics, 164, 1279-1289.

[7] Rocha, E.P.C., Danchin, A. and Viari, A. (1999) Functional and Evolutionary Roles of Long Repeats in Prokaryotes. Research in Microbiology, 150, 725-733. http://dx.doi.org/10.1016/S0923-2508(99)00120-5 
[8] Shapiro, J.A. and von Sternberg, R. (2005) Why Repetitive DNA Is Essential to Genome Function. Biological Review, 80, 227-250. http://dx.doi.org/10.1017/S1464793104006657

[9] van Belkum, A. (1999) Short Sequence Repeats in Microbial Pathogenesis and Evolution. Cellular and Molecular Life Sciences, 56, 729-734. http://dx.doi.org/10.1007/s000180050019

[10] van Belkum, A., Scherer, S., van Alphen, L. and Verbrugh, H. (1998) Short-Sequence DNA Repeats in Prokaryotic Genomes. Microbiology and Molecular Biology Reviews, 62, 275 293.

[11] Mojica, F.J., Diez-Villasenor, C., Soria, E. and Juez, G. (2000) Biological Significance of a Family of Regularly Spaced Repeats in the Genomes of Archaea, Bacteria and Mitochondria. Molecular Microbiology, 36, 244-246. http://dx.doi.org/10.1046/j.1365-2958.2000.01838.x

[12] Doudna, J.A. and Charpemtier, E. (2014) Genome Editing: The New Frontier of Genome Engineering with CRISPR-Cas 9. Science, 346, 1258096.

[13] Jansen, R., Embden, J.D., Gaastra, W. and Schouls, L.M. (2002) Identification of Genes That Are Associated with DNA Repeats in Prokaryotes. Molecular Microbiology, 43, 1565-1575. http://dx.doi.org/10.1046/j.1365-2958.2002.02839.x

[14] Bland, C., Ramsey, T.L., Sabree, F., Lowe, M., Brown, K., Kyrpides, N.C. and Hugenholtz, P. (2007) CRISPR Recognition Tool: A Tool for Automatic Detection of Clusetered Regularly Interspaced Palidromic Repeats. BMC Bioinformatics, 8, 209. http://dx.doi.org/10.1186/1471-2105-8-209

[15] Benson, G. (1999) Tandem Repeats Finder: A Program to Analyze DNA Sequences. Nucleic Acids Research, 27, 573-580. http://dx.doi.org/10.1093/nar/27.2.573

[16] Grissa, I., Vergnand, G. and Pourcel, C. (2007) CRISPRFinder: A Web Tool to Identify Clustered Regularly Interspaced Short Palindromic Repeats. Nucleic Acids Research, 35, 52-57. http://dx.doi.org/10.1093/nar/gkm360

[17] Funkhouser, S. (2013) General Formulation for the Entropy of A Graph or Probability Matrix. Unpublished Paper, SPAWAR, SC.

Submit or recommend next manuscript to SCIRP and we will provide best service for you:

Accepting pre-submission inquiries through Email, Facebook, LinkedIn, Twitter, etc.

A wide selection of journals (inclusive of 9 subjects, more than 200 journals)

Providing 24-hour high-quality service

User-friendly online submission system

Fair and swift peer-review system

Efficient typesetting and proofreading procedure

Display of the result of downloads and visits, as well as the number of cited articles

Maximum dissemination of your research work

Submit your manuscript at: http://papersubmission.scirp.org/

Or contactcmb@scirp.org 